\title{
Modeling odor responses of projection neurons and Kenyon cells in insects
}

\author{
Paul Pawletta ${ }^{*}$, Amir Madany Mamlouk \\ From 1st International Workshop on Odor Spaces \\ Hannover, Germany. 4-7 September 2013
}

Odorants are chemical substances which evoke certain odors in the brain for example fruity or nutty. Hence, it should be possible to predict the smell of an odorant from its chemical structure. However, this task is not easy because each odorant encodes many odors and chemical compositions of odorants are often similar. The olfactory system of insects is well suited for a better understanding of odor processing. In locusts antennal lobe are about 90000 receptor neurons where odorants can bind. The second neuron layer consists of 800 projection neurons (PNs) that receives input from the receptor neurons and sends its signals to the Kenyon cell layer (KCs). In locusts there are about 50,000 KCs and each $\mathrm{KC}$ is connected with an approximate probability 0.5 with each PN. Furthermore the outgoing signal of the KC-layer is sparse, only $2 \%$ spiking KCs on average [1].

For modeling the locust olfactory system in MATLAB we used 836 odorants from the Sigma- Aldrich Flavors and Fragrances Catalogue. Each odorant is described by 184 chemical properties and at least one odor descriptor. The PN-layer is modeled with a self-organizing map [2] consisting of 800 neurons to mimic the locust olfactory system. For all odorants the response of the PNs can be expressed as a vector. Every element of the vector represents a single $\mathrm{PN}$ and its activation is expressed by a numerical value between 0 (no activation) and 1 (maximal activation). This vector serves as the input for the $\mathrm{KC}$-layer. We assumed the connection probability between $\mathrm{KCs}$ and PNs as random and normally distributed and calculated $\mathrm{KC}$ responses for different values of connection probability and variance. In the last step, a firing threshold is implemented that allows only a few KCs to fire a spike [3].

To compare the activity patterns of PNs and KCs we considered a binary classification problem. Each odorant belongs to one odor class (e.g. fruity) or not (e.g. not fruity). The classification is done by a hard-margin support vector machine with a linear kernel function. For 16 odor descriptors the performance of the classification is assessed by the Accuracy (Acc) and the Matthews correlation coefficient (MCC). For modeling the locust olfactory system, the PN-layer was modeled with 800 neurons and the KC-layer with 50000 neurons. The results for the classification of the PN- responses yield a mean Accuracy of $74 \%$ and a mean MCC of 0.12 . While the classification of the $\mathrm{KC}$-response without a firing threshold slightly improves classification performance $(\mathrm{Acc}=76 \%, \mathrm{MCC}=$ 0.13), KC-responses with particular values for connection probability and firing threshold outperform PN-responses $($ Acc $=89 \%, M C C=0.3)$. These results suggest that the response patterns of $\mathrm{KCs}$ are better linear separable then the responses of PNs.

Published: 16 April 2014

\section{References}

1. Cassenaer S, Laurent G: Hebbian STDP in mushroom bodies facilitates the synchronous flow of olfactory information in locusts. Nature 2007, 448:709-713.

2. Schmuker M, Schneider G: Processing and classification of chemical data inspired by insect olfaction. Proc Natl Acad Sci 2007, 104:20285-20289.

3. Jortner RA: Network architecture underlying maximal separation of neuronal representations. Front Neuroeng 2012, 5:19.

doi:10.1186/2044-7248-3-S1-P13

Cite this article as: Pawletta and Mamlouk: Modeling odor responses of projection neurons and Kenyon cells in insects. Flavour 2014 3(Suppl 1): P13. 